\title{
Information Integration Design and Implementation of Satellite Communication Earth Station Central Station
}

\author{
Lv Zhibo, a , Li Ziyü, b, Liu Yan ${ }^{1, c}$ and Kong Deyang ${ }^{1, d}$ \\ ${ }^{1}$ Beijing Aerospace Control Center, Beijing 102206, China; \\ alzb0099@163.com, ${ }^{b}$ licctv8848@gmail.com, 'vivianliuyan@gmail.com, ${ }^{d}$ leok126@126.com
}

Keywords: Information Integration, Central Station, Satellite Communication.

\begin{abstract}
From the perspective of information integration, as an example, this paper implements the integration of the central station of a satellite communication network, both horizontally and vertically. It proceeds from the satellite communication terminal equipments, redesigns connection between interfaces, as well as condition monitoring system and channel monitoring system; it fixes hardware connection between machine rooms. This central station completes its work through software and information platform.
\end{abstract}

\section{Introduction}

Information integration has been widely used in various industries. Satellite communication is no exception [1]. Domestic satellite earth stations have done some related work, but far from satisfying demands of daily work. Information integration of satellite earth station means unified extensible interface platform horizontally, as well as monitoring system ensuring accurate information transmission vertically. This paper implements the integration of the central station of a satellite communication network both horizontally and vertically. It provides a reference for the implementation of other satellite communication earth stations.

Central station of satellite communication earth station is the central nervous of the satellite communication network. As business expands and the number of equipments increases, a series of problems arise: First, original planning is broken and layout needs to be adjusted according to actual situation, which makes machine room layout unreasonable; Second, cable distribution is implemented by different factories, which result in cable overlapping, and makes cable cleaning very difficult. Third, as business increases, single satellite communication link equipment services several users, and equipment connection needs to be adjusted frequently. The service life of cable connector is 500 times in general, which makes it necessary to prolong service life of connectors. Last but not least, due to lack of monitoring method, low reliability, and bad expandability of old monitoring systems, reliability evaluation of the whole network becomes very difficult. Above-mentioned problems must be solved to fundamentally promote construction of satellite earth station.

\section{Hardware System Design and Implementation}

Communication earth station machine rooms are generally divided into radio frequency machine rooms and terminal equipment rooms. RF rooms are usually near the antennas, while Terminal equipment room is the main place where operators work and operate equipments. Therefore, machine rooms can be separated as Equipment zone and the workspace. Aiming at the current situation that large amount and type of equipments were in the terminal rooms, this paper redesigns and installs equipments such as modems and routers in the terminal rooms.

Modularization and Standardization of Equipment Installation. We decompose communication process and modularize link parts. Sub modules at the same level back up for each other [2]; superior and subordinate modules are connected by switching systems; a parallel mesh structure is composed, which increases communication reliability. Data interface within each module employs standardized design, and data in each equipment can be transmitted to any user in the 
network. When user business changes, cable doesn't have to be readjusted. Non-uniform cable distribution is avoided, and machine room standardization is improved. See Figure.1.

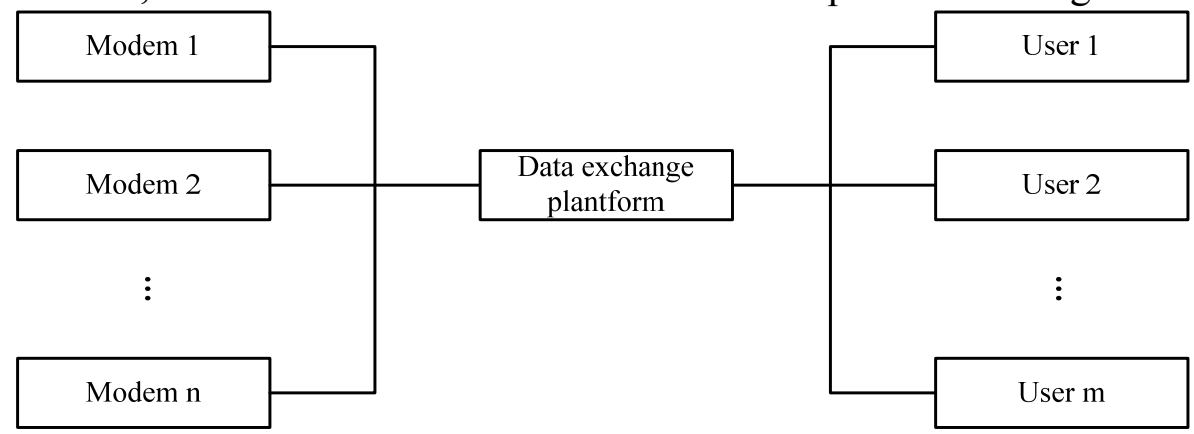

Fig.1 Standardization of data interface connection diagram

Satcom Link Signal Process Automatic Control and Seamless Switching Technology. Through the modular design and a new intermediate frequency switching system, flexible transformation of various types of lines is achieved. In the test, switching signal to local operation can be completed in 10 seconds and remote operation can be done in 5 seconds. Flexibility and fairness of the intermediate frequency signal transmission is enhanced, and the efficiency of business processing is improved. Satcom link automatic adjustment is implemented, which means, any communication link in the center station can establish a communication circuit with any user station within the network, without any hardware adjustments. See figure.2.

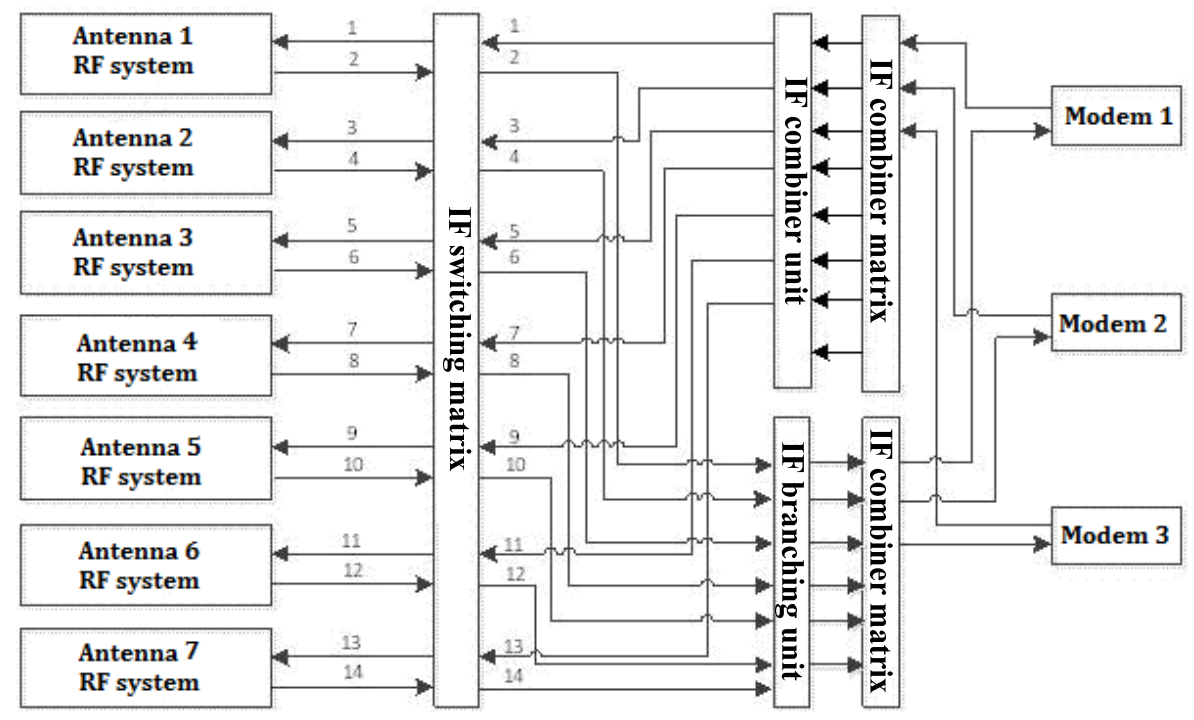

Fig.2 Intermediate frequency signal interface connection diagram

\section{Software System Design and Implementation}

Central station software system mainly includes three layers [3]: the equipment layer, the information layer and the display layer. The equipment layer mainly realize the united monitoring and management of all equipments and frequency resources of the entire network; the information layer monitors spectrum information of the entire network and provides alarm function [4]; the display layer integrates monitoring a variety of software and display according to work demands.

Design and Implementation of Multi-station Hierarchical Remote Monitoring and Controlling System. Hierarchically, user stations are divided into central station, regional station and remote station, forming a three-level management system structure. First-level stations (central stations) implement management through second-level stations (regional stations), which is to manage and allocate frequency resources of the whole network as well as to remotely monitor and control equipments of third-level stations (remote stations). Second-level stations manage stations within their respective regions, in charge of management of equipments and resources. Third-level 
stations monitor and control local Satcom equipments. New equipments can be directly added to the system through the software interface, without having to rewrite code and software design. Equipments in the network can be monitored and controlled through the software.

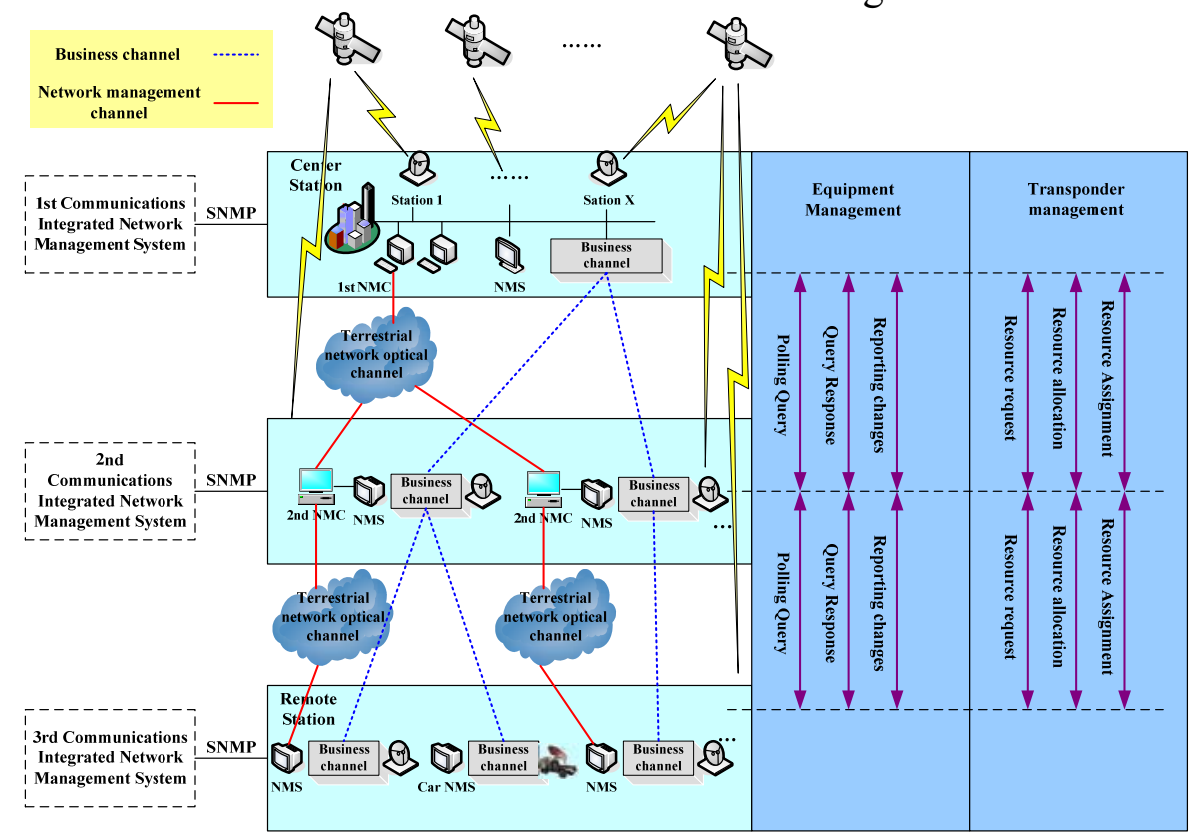

Fig.3 Network management center connection diagram

Design and Implementation of Integrated Real-time Spectrum Monitoring System. Since our work raises high requirements for real-time spectrum, we developed real-time spectrum analysis software based on fast Fourier transform (FFT) [5]. The real-time spectrum monitoring system is composed by real-time spectrum information collecting equipment, high-speed transmission equipment, disk array server and the corresponding application software. Main function of information collecting equipment is to real-time sample and process satellite signal spectrum information, which consist of sampling and processing spectrum, data forwarding and storage, exception alarm and etc; high-speed transmission equipment shares and transmits spectrum information of each detection module. Software includes interface software module, data storage module, communication module of external application software, analyzing and processing module, interface display module, and business query module. 24-hour continuous monitoring of transmission signal and a real-time alarm on abnormal signal state and disturbance signal, improve the abnormal signal processing efficiency of the central station [6]. See figure.4.

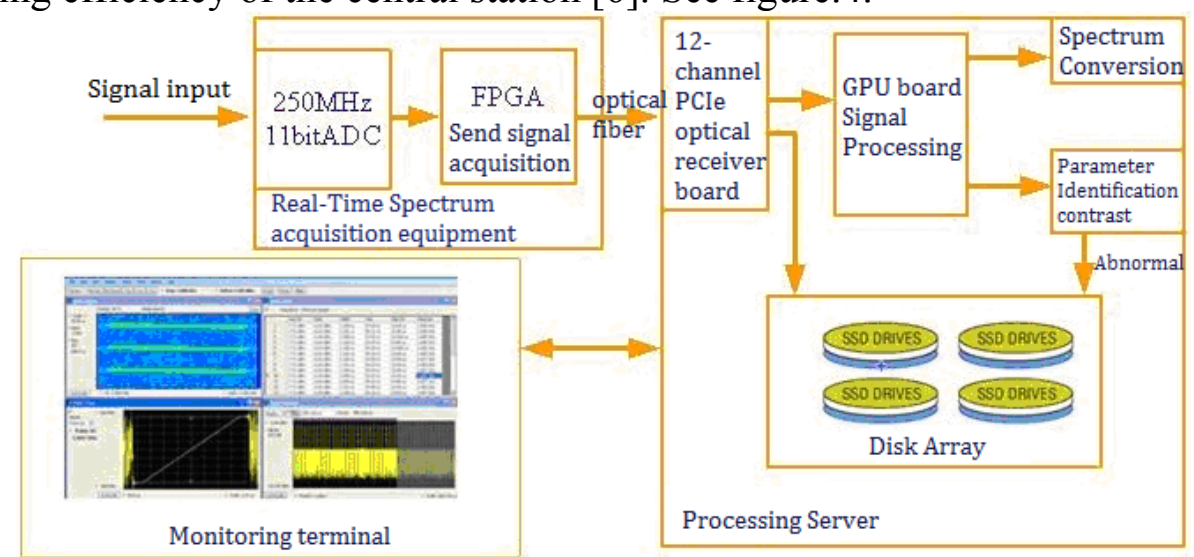

Fig.4 Integrated real-time spectrum monitoring system schematic diagram

Design and Implementation of Integrated Display System. Signal sources such as equipment working parameters, frequency spectrum and video image are the major means of operators to monitor technical state of equipments, which are also used to judge whether communication link is normal or not. Signal source is modulated, and sent to the public information display platform, where 
KVM switches different sources. Operators may access new sources, as well as display and switch according to demands. It support a maximum of 64 inputs and 16 outputs. See figure.5.

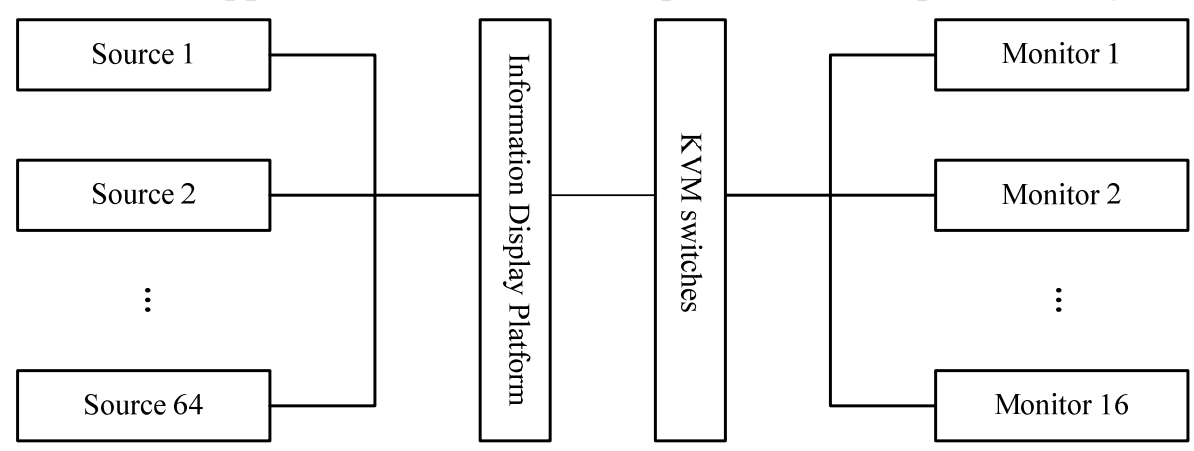

Fig.5 integrated information display platform connection diagram

\section{Conclusion}

By studying the characteristics of information integration, this paper carefully analyzes actual work situation of satellite communication earth station. It redesigns hardware and software systems of the central station, fixes hardware connections between machine rooms, which make business adjustments more convenient. This paper fulfills software functions, as well as strengthens the software extensibility, which effectively solves the problem arises when a variety of similar software running in the same grid, and promotes the integration of satellite communication earth station information.

\section{References}

[1] Akyildiz, I. F., \& Jeong, S. H. (1997). Satellite ATM networks: A survey. Communications Magazine, IEEE, 35(7), 30-43.

[2] Xiao, X., Fu, Z., Liu, G., \& Deng, C. (2010, October). A backup data network for power system automations based on satellite communication. In Power System Technology (POWERCON), 2010 International Conference on (pp. 1-5). IEEE.

[3] Di, W., \& Qing, L. (2005, October). A new routing algorithm of two-tier LEO/MEO mobile satellite communication systems. In Communications, 2005 Asia-Pacific Conference on (pp. 111-115). IEEE.

[4] Wenbo, Z., \& Qian, D. (2010, September). A Fault Diagnosis Method in Satellite Networks. In Wireless Communications Networking and Mobile Computing (WiCOM), 2010 6th International Conference on (pp. 1-4). IEEE.

[5] Kschischang, F. R., Frey, B. J., \& Loeliger, H. A. (2001). Factor graphs and the sum-product algorithm. Information Theory, IEEE Transactions on, 47(2), 498-519.

[6] Wang, H., Liu, G., Ge, X., \& Mao, X. (2011, August). Performance evaluation and capacity analysis for IDMA-based satellite communication system. In Communications and Networking in China (CHINACOM), 2011 6th International ICST Conference on (pp. 604-608). IEEE. 